\title{
FUNDAMENTAL SOLUTIONS FOR A CLASS OF HYPOELLIPTIC PDE GENERATED BY COMPOSITION OF QUADRATIC FORMS
}

\author{
BY \\ AROLDO KAPLAN ${ }^{1}$
}

\begin{abstract}
We introduce a class of nilpotent Lie groups which arise naturally from the notion of composition of quadratic forms, and show that their standard sublaplacians admit fundamental solutions analogous to that known for the Heisenberg group.
\end{abstract}

By a theorem of Hormander [6], if $X_{1}, \ldots, X_{l}$ are vector fields on a manifold $N$ with the property that their commutators up to a certain order span the tangent space at every point, then the differential operator

$$
D=\sum_{j} X_{j}^{2}
$$

is hypoelliptic; that is, the solutions of the equation $D f=g$ with $g \in C^{\infty}$, are also $C^{\infty}$. Especially interesting is the case where $N$ is a nilpotent Lie group and the $X_{j}$ 's are generators of its Lie algebra; in particular, these "sublaplacians" play a central role in the Rothschild-Stein regularity theory of second order hypoelliptic equations [9].

In this paper we introduce a class of step-2 nilpotent groups (type $\mathrm{H}$ ) whose standard sublaplacians are shown to admit explicit fundamental solutions of an elementary form. This phenomenon had been originally observed in the case of the Heisenberg group by Folland [4] (cf. also [7]). For the class introduced here, heuristic evidence suggests that it should be "the largest" with that property; in any case, it yields the previously known examples together with infinitely many new ones. The analytic hypoellipticity of the differential operators involved follows as an immediate consequence of the formula for their fundamental solution.

Groups of type $\mathrm{H}$ arise in a natural manner from the so-called compositions of quadratic forms (or "orthogonal multiplications"), a notion that has found various applications in algebra and topology [2], [3], [8]; we use a classical result from this theory to give a measure of the size of the class of sublaplacians so obtained. Besides, the relationship between that notion and nilpotent groups may have some interest on its own.

Independently, B. Helffer found similar solutions for a class of groups that turned out to be equivalent to ours (personal communication). Finally, we wish to thank L. Rothschild and the reviewer for their useful suggestions, in particular those concerning the question of analytic hypoellipticity discussed at the end of $\$ 2$.

Received by the editors February 21, 1979.

AMS (MOS) subject classifications (1970). Primary 35H05, 22E30; Secondary 10C05, 22 E25.

${ }^{1}$ Partially supported by an NSF grant. 
1. Lie algebras of type $\mathrm{H}$ and composition of quadratic forms. All vector spaces will be assumed real and finite-dimensional. A Lie algebra $\mathfrak{n}$ is 2-step nilpotent if $[\mathfrak{n},[\mathfrak{n}, \mathfrak{n}]]=\{0\}$; if $z$ is the center of $\mathfrak{n}$ and $\mathfrak{b}$ any linear complement, the Lie bracket can be regarded as a vector-valued skew-symmetric bilinear form

$$
[,]: \mathfrak{v} \times \mathfrak{v} \rightarrow \mathfrak{z}
$$

which is "nondegenerate", in the sense that $[v, \mathfrak{v}]=0 \Rightarrow v=0$. If $\operatorname{dim} z=1$, there is (up to isomorphism) only one such Lie algebra of each odd dimension, namely the Heisenberg algebra. The Lie bracket can there be regarded as a standard real valued, nondegenerate skew form on $\mathfrak{b}$. If $J: \mathfrak{b} \rightarrow \mathfrak{b}$ is any complex structure leaving that form invariant

$$
J^{2}=-I, \quad\left[J v, J v^{\prime}\right]=\left[v, v^{\prime}\right]
$$

then $\left\langle v, v^{\prime}\right\rangle=\left[J v, v^{\prime}\right]$ defines a symmetric form on $\mathfrak{v}$ which can be made positive definite by an appropriate choice of $J$. It is easy to see that under this inner product, the map $\operatorname{ad}_{v}: \mathfrak{v} \rightarrow z, \operatorname{ad}_{v}\left(v^{\prime}\right)=\left[v, v^{\prime}\right]$ identifies isometrically the orthogonal complement of its kernel with $z \approx \mathbf{R}$. This motivates the following definition.

Let $\boldsymbol{n}$ be a 2-step nilpotent algebra endowed with an inner product $\langle$,$\rangle and let \mathfrak{v}$ be the orthogonal complement to its center $z$. For each $v \in \mathfrak{v}$ consider the orthogonal decomposition

$$
\mathfrak{v}=\mathfrak{l}_{v} \oplus \mathfrak{v}_{v}
$$

where $\mathfrak{f}_{v}=\operatorname{ker}\left(\operatorname{ad}_{v}: \mathfrak{b} \rightarrow z\right)=\left\{v^{\prime} \in \mathfrak{b}:\left[v, v^{\prime}\right]=0\right\}$. We shall say that $\mathfrak{n}$ is of type $\mathrm{H}$ if

$$
\operatorname{ad}_{v}: \mathfrak{b}_{v} \rightarrow z \text { is a surjective isometry for every unit vector } v \in \mathfrak{b} .
$$

We now recall the definition of composition of quadratic forms [8]. Let $\mathfrak{u}, \mathfrak{v}$ be vector spaces each equipped with a positive definite quadratic form ||$^{2}$. A composition of these quadratic forms is a bilinear map

$$
\mu: \mathfrak{u} \times \mathfrak{v} \rightarrow \mathfrak{b}
$$

satisfying

$$
|\mu(u, v)|=|u||v| \quad \text { for all } u \in \mathfrak{u}, v \in \mathfrak{v} .
$$

We shall assume that these $\mu$ 's are normalized in the sense that $\mu\left(u_{0}, v\right)=v$ for some $u_{0} \in \mathfrak{u}$. Such normalization can always be achieved by choosing an arbitrary unit vector $u_{0} \in \mathfrak{u}$ and redefining $\mu^{\prime}(u, v)=\mu\left(u, T^{-1}(v)\right)$, where $T: \mathfrak{b} \rightarrow \mathfrak{b}$ is $T(v)=\mu\left(u_{0}, v\right)$.

Starting with a composition of quadratic forms, one can construct a Lie algebra of type $\mathrm{H}$ as follows. Define $\Phi: \mathfrak{v} \times \mathfrak{v} \rightarrow \mathfrak{u}$ by demanding

$$
\left\langle u, \phi\left(v, v^{\prime}\right)\right\rangle=\left\langle\mu(u, v), v^{\prime}\right\rangle, \quad u \in \mathfrak{u} ; v, v^{\prime} \in \mathfrak{v},
$$

relative to the inner products induced by the given quadratic forms. Let $z$ denote the orthogonal complement to $\mathbf{R} u_{0}$ in $\mathfrak{u}$ and $\pi: \mathfrak{u} \rightarrow \mathfrak{z}$ the orthogonal projection. Now set

$$
\mathfrak{n}=\mathfrak{v} \times \mathfrak{z}
$$


and define a bracket on $\mathfrak{n}$ by

$$
\left[\left(v_{1}, z_{1}\right),\left(v_{2}, z_{2}\right)\right]=\left(0, \pi \circ \phi\left(v_{1}, v_{2}\right)\right) .
$$

THEOREM 1. $\mathfrak{n}$, equipped with the above bracket and the obvious inner product, is a Lie algebra of type H. Moreover, any such Lie algebra arises in this manner.

Proof. While developing the argument we shall record some identities that will be needed in the next section. Each $u \in \mathfrak{u}$ defines a linear map $J_{u}: \mathfrak{b} \rightarrow \mathfrak{b}$ by

$$
J_{u}(v)=\mu(u, v)
$$

which obviously satisfies $\left|J_{u}(v)\right|=|u||v|$. Together with the linearity of $u \rightarrow J_{u}$ this yields

$$
\left\langle J_{u}(v), J_{u^{\prime}}(v)\right\rangle=\left\langle u, u^{\prime}\right\rangle|v|^{2} .
$$

Setting $u^{\prime}=u_{0}, u=z \in z$, one obtains

$$
\left\langle J_{z}(v), v\right\rangle=0 \text { for } z \in z, v \in \mathfrak{v},
$$

implying that $J_{z}$ is skew-adjoint. From

$$
\left\langle z, \phi\left(v, v^{\prime}\right)\right\rangle=\left\langle J_{z}(v), v^{\prime}\right\rangle
$$

it now follows that the bilinear map $\pi \circ \phi: \mathfrak{b} \times \mathfrak{b} \rightarrow \mathfrak{z}$ is skew-symmetric. Therefore $\mathfrak{n}$ is a Lie algebra which is clearly step- 2 nilpotent and whose center contains $z$.

Another consequence of (7) is that $J_{z}(v)$ is always orthogonal to the kernel of $\operatorname{ad}_{v}: \mathfrak{v} \rightarrow \mathfrak{z}$, that is

$$
J_{z}(v) \in \mathfrak{v}_{v} .
$$

Indeed, $J_{z}(v)$ is the (necessarily unique) element in $\mathfrak{b}_{v}$ such that

$$
\left[v, J_{z}(v)\right]=|v|^{2} z
$$

(we are using here the identification $\mathfrak{n}=\mathfrak{b} \times \mathfrak{z} \sim \mathfrak{v} \oplus \mathfrak{z}$ ), as can be seen from the identity

$$
\left\langle z^{\prime},\left[v, J_{z}(v)\right]\right\rangle=\left\langle J_{z^{\prime}}(v), J_{z}(v)\right\rangle=\left\langle z^{\prime}, z\right\rangle|v|^{2} .
$$

From (8) and (9) one deduces, in the first place, that $\mathfrak{v}$ contains no nonzero element from the center of $\mathfrak{n}$, so that the latter coincides with $z$; and secondly (recalling $\left.\left|J_{z}(v)\right|=|z||v|\right)$ that the map

$$
\operatorname{ad}_{v}: \mathfrak{b}_{v} \rightarrow \mathfrak{z}
$$

is a surjective isometry whenever $|v|=1$. Thus $\mathfrak{n}$ is a Lie algebra of type $\mathrm{H}$ as asserted.

Suppose now that $\mathfrak{n}$ is a Lie algebra of type $H$, with its standand decomposition $\mathfrak{n}=\mathfrak{v} \oplus \mathfrak{z}$. Then, for each $z \in \mathfrak{z}$, the bilinear form $v, v^{\prime} \rightarrow\left\langle z,\left[v, v^{\prime}\right]\right\rangle$ on $\mathfrak{b}$ is skew-symmetric and nondegenerate for $z \neq 0$; therefore there exist a linear isomorphism $J_{z}: \mathfrak{v} \rightarrow \mathfrak{b}$ satisfying

$$
\begin{aligned}
& \text { (i) }\left\langle J_{z}(v), v^{\prime}\right\rangle=\left\langle z,\left[v, v^{\prime}\right]\right\rangle, \\
& \text { (ii) }\left\langle J_{z}(v), v^{\prime}\right\rangle+\left\langle v, J_{z}\left(v^{\prime}\right)\right\rangle=0
\end{aligned}
$$


for $v, v^{\prime} \in \mathfrak{b}, z \in \mathfrak{z}$. We now claim that the identities (5), (8) and (9) are valid in the present context as well. Indeed, (8) follows immediately from (10)(i). Let $0 \neq v \in$ $\mathfrak{b}, z \in z$; then $(\mathrm{H})$ assures the existence of a (unique) $v^{\prime} \in \mathfrak{b}_{v}$ such that $\left[v, v^{\prime}\right]=z$; moreover, since $\operatorname{ad}_{|v|^{-1} v}: \mathfrak{b}_{v} \rightarrow \mathfrak{z}$ must be an isometry, one has that for any $v^{\prime \prime} \in \mathfrak{b}_{v}$,

$$
|v|^{2}\left\langle v^{\prime}, v^{\prime \prime}\right\rangle=\left\langle\left[v, v^{\prime}\right],\left[v, v^{\prime \prime}\right]\right\rangle=\left\langle z,\left[v, v^{\prime \prime}\right]\right\rangle=\left\langle J_{z}(v), v^{\prime \prime}\right\rangle
$$

(notice that the above identities hold as long as either $v^{\prime}$ or $v^{\prime \prime}$ lie in $\mathfrak{v}_{v}$ ). Therefore $J_{z}(v)=|v|^{2} v^{\prime}$, showing (9). Finally, let $z, z^{\prime} \in z, v \in \mathfrak{v}$; from (10)(i) and (9) one gets $\left\langle J_{z}(v), J_{z^{\prime}}(v)\right\rangle=\left\langle z,\left[v, J_{z^{\prime}}(v)\right]\right\rangle=|v|^{2}\left\langle z, z^{\prime}\right\rangle$, giving (5) (for $u, u^{\prime} \in$ z).

Polarizing (5) one obtains

$$
\left\langle J_{z}(v), J_{z^{\prime}}\left(v^{\prime}\right)\right\rangle+\left\langle J_{z^{\prime}}(v), J_{z}\left(v^{\prime}\right)\right\rangle=2\left\langle v, v^{\prime}\right\rangle\left\langle z, z^{\prime}\right\rangle
$$

which, because of (10)(ii), yields

$$
J_{z} J_{z^{\prime}}+J_{z^{\prime}} J_{z}=-2\left\langle z, z^{\prime}\right\rangle I
$$

In particular,

$$
J_{z}^{2}=-|z|^{2} I
$$

But, since $J_{z}$ is also skew-adjoint, one gets

$$
\left|J_{z}(v)\right|=|z||v| .
$$

Set now $\mathfrak{u}=z \oplus \mathbf{R}$ with the obvious inner product and define a bilinear $\mu$ : $\mathfrak{u} \times \mathfrak{v} \rightarrow \mathfrak{u}$ by

$$
\mu(z+\lambda, v)=J_{z}(v)+\lambda v \quad(z \in z, v \in \mathfrak{v}, \lambda \in \mathbf{R}) .
$$

Then (13) shows that $\mu$ is a composition of the corresponding quadratic forms. This concludes the proof of the theorem.

The following consequence is interesting from the point of view of the results of the next section. Let $\rho$ be the function defined on the nonnegative integers by

$$
\text { if } n=(\text { odd }) 2^{4 p+q}, 0 \leqslant q<3 \text {, then } \rho(n)=8 p+2^{q} \text {. }
$$

A classical theorem of Hurwitz, Radon and Eckmann [3] gives a necessary and sufficient condition for the existence of a composition of quadratic forms $\mu$ : $\mathfrak{u} \times \mathfrak{v} \rightarrow \mathfrak{v}$ : it is that $0 \leqslant \operatorname{dim} \mathfrak{u} \leqslant \rho(\operatorname{dim} \mathfrak{v})$. We therefore have the

COROLlaRY 1. Let $0<m<n$ be integers. Then there exist an $n$-dimensional Lie algebra of type $\mathrm{H}$ with $m$-dimensional center if and only if $m<\rho(n-m)$. In particular, there are type $\mathrm{H}$ Lie algebras with centers of any given dimension.

2. Sublaplacians and their fundamental solutions. Let $\mathfrak{n}$ be a Lie algebra of type $\mathbf{H}$ and $N$ the corresponding simply connected analytic group. Since the subspace $\mathfrak{b}$ in

$$
\mathfrak{n}=\mathfrak{v} \oplus \mathfrak{z}
$$

generates $\mathfrak{n}$, the second order differential operator

$$
D=\sum_{j} X_{j}^{2}, \quad\left\{X_{j}\right\}=\text { orthogonal basis of } \mathfrak{v},
$$

is hypoelliptic on $N$. Notice that $D$ is invariant not only under left-translations, but also under those automorphisms of $N$ whose differentials preserve the given inner product. 
The exponential mapping of a simply connected Lie group is an analytic diffeomorphism. One can then define analytic mappings $v: N \rightarrow \mathfrak{b}, z: N \rightarrow \mathrm{z}$ by

$$
n=\exp (v(n)+z(n)), \quad n \in N \text {. }
$$

THEOREM 2. There exist a constant $c$ such that the function

$$
\Phi(n)=c\left(|v(n)|^{4}+16|z(n)|^{2}\right)^{-k},
$$

$R=\frac{1}{4}(\operatorname{dim} \mathfrak{n}+\operatorname{dim} z-2)$, is a fundamental solution for the operator $D$.

Proof. By analogy with Folland's argument for the case of Heisenberg group, we consider the one-parameter family

$$
\Phi_{\varepsilon}(n)=c\left(\left(|v(n)|^{2}+\varepsilon^{2}\right)^{2}+16|z(n)|^{2}\right)^{-k}
$$

These functions are everywhere analytic for $\varepsilon \neq 0$, and $\Phi_{0}=\Phi$. Fix a point $n \in N$ and set

$$
\phi_{j}(t)=\left(\left|v\left(n \exp t X_{j}\right)\right|^{2}+\varepsilon^{2}\right)^{2}+16\left|z\left(n \exp t X_{j}\right)\right|^{2}, \quad j=1, \ldots, l ;
$$

since $\phi_{j}(0)$ is independent of $j$ we denote it simply by $\phi(0)$. Then

$$
\begin{aligned}
X_{j}^{2} \Phi_{e}(n) & =\left.\frac{d^{2}}{d t^{2}} \Phi_{e}\left(n \exp t X_{j}\right)\right|_{t=0}=c \frac{d^{2}}{d t^{2}} \phi_{j}(t)^{-k} \\
& =c k \phi(0)^{-k-2}\left((k+1)\left(\phi_{j}^{\prime}(0)\right)^{2}-\phi(0) \phi_{j}^{\prime \prime}(0)\right) .
\end{aligned}
$$

The formula $\exp x \exp y=\exp \left(x+y+\frac{1}{2}[x, y]\right)$ holds in any step-2 nilpotent group. In particular, $v\left(n \exp t X_{j}\right)=v(n)+t X_{j}, z\left(n \exp t X_{j}\right)=z(n)+$ $(t / 2)\left[v(n), X_{j}\right]$ and one gets

$$
\begin{aligned}
\phi_{j}(t) & =\left(\left|v(n)+t X_{j}\right|^{2}+\varepsilon^{2}\right)^{2}+16\left|z(n)+(t / 2)\left[v(n), X_{j}\right]\right|^{2}, \\
\phi_{j}^{\prime}(0) & =4\left(\left(|v(n)|^{2}+\varepsilon^{2}\right)\left\langle v(n), X_{j}\right\rangle+4\left\langle z(n),\left[v(n), X_{j}\right]\right\rangle\right), \\
\phi_{j}^{\prime \prime}(0) & =4\left(2\left\langle v(n), X_{j}\right\rangle^{2}+\left|X_{j}\right|^{2}\left(|v(n)|^{2}+\varepsilon^{2}\right)+2\left|\left[v(n), X_{j}\right]\right|^{2}\right) .
\end{aligned}
$$

In the notation of $\S 1, \phi_{j}^{\prime}(0)$ can be written as $4\left\langle\left(|v(n)|^{2}+\varepsilon^{2}\right) v(n)+\right.$ $4 J_{z(n)}(v(n)), X_{j}>$ and since $\left\{X_{j}\right\}$ is an orthonormal basis of $\mathfrak{b},(5)$ and (8) yield

$$
\begin{aligned}
\sum_{j}\left(\phi_{j}^{\prime}(0)\right)^{2} & =16\left|\left(|v(n)|^{2}+\varepsilon^{2}\right) v(n)+4 J_{z(n)}(v(n))\right|^{2} \\
& =16\left(\left(|v(n)|^{2}+\varepsilon^{2}\right)^{2}|v(n)|^{2}+16|v(n)|^{2}|z(n)|^{2}\right) \\
& =16|v(n)|^{2} \phi(0) .
\end{aligned}
$$

Let now $\left\{z_{i}\right\}$ be any orthonormal basis of $z$; then

$$
\begin{aligned}
\sum_{j}\left|\left[v(n), X_{j}\right]\right|^{2} & =\sum_{i} \sum_{j}\left\langle z_{i},\left[v(n), X_{j}\right]\right\rangle^{2} \\
& =\sum_{i} \sum_{j}\left\langle J_{z_{i}}(v(n)), X_{j}\right\rangle^{2}=\sum_{i}\left|J_{z_{i}}(v(n))\right|^{2} \\
& =\sum_{i}\left|z_{i}\right|^{2}|v(n)|^{2}=(\operatorname{dim} z)|v(n)|^{2},
\end{aligned}
$$


and therefore

$$
\sum_{j} \phi_{j}^{\prime \prime}(0)=4\left((2+\operatorname{dim} \mathfrak{v}+2 \operatorname{dim} z)|v(n)|^{2}+\varepsilon^{2} \operatorname{dim} \mathfrak{z}\right) .
$$

With $k=\frac{1}{4}(\operatorname{dim} \mathfrak{n}+\operatorname{dim} z-2),(14),(15)$ and (16) now give

$$
D \Phi_{\varepsilon}(n)=-4(\operatorname{dim} \mathfrak{v}) c k \varepsilon^{2}\left(\left(|v(n)|^{2}+\varepsilon^{2}\right)^{2}+16|z(n)|^{2}\right)^{-k-1} .
$$

In particular,

$$
D \Phi \equiv 0 \text { away from the identity. }
$$

Under the one-parameter group of automorphisms of $N$

$$
\varepsilon \rightarrow \delta_{e}(n)=\exp \left(t v(n)+t^{2} z(n)\right)
$$

the Haar measure $d n$ induced by the ordinary Lebesgue measure in $\mathfrak{n}$ transforms like

$$
\delta_{\varepsilon}(d n)=\varepsilon^{4 k+2} d n
$$

moreover,

$$
\left(D \Phi_{\varepsilon}\right) \circ \delta_{\varepsilon}=\varepsilon^{-4 k-2} D \Phi_{1} .
$$

The rest of the argument is standard: because $k+1>\frac{1}{4} \operatorname{dim} \mathfrak{v}+\frac{1}{2} \operatorname{dim} z$, the function $D \Phi_{\varepsilon}$ is integrable for every $\varepsilon>0$ and it is clear that

$$
\int_{N(\lambda)}\left(D \Phi_{\varepsilon}\right)(n) d n=\int_{N\left(\lambda / \varepsilon^{2}\right)}\left(D \Phi_{1}\right)(n) d n
$$

where we have set $N(\lambda)=\left\{n \in N:|v(n)|^{4}+16|z(n)|^{2}>\lambda\right\}$. Thus

$$
\lim _{\varepsilon \rightarrow 0} \int_{N(\lambda)}\left(D \Phi_{\varepsilon}\right)(n) d n=0 \text { for all } \lambda>0 .
$$

If the constant $c$ is chosen so as to make $\int_{N}\left(D \Phi_{1}\right)(n) d n=1$, the left-invariance of $D$ implies now

$$
D(f * \Phi)=\lim _{\varepsilon \rightarrow 0} D\left(f * \Phi_{\varepsilon}\right)=\lim _{\varepsilon \rightarrow 0} f * D \Phi_{\varepsilon}=f
$$

for any smooth function $f$ with compact support. This finishes the proof of the theorem.

COROLlaRY 2. The operator $D$ is analytically hypoelliptic.

L. Rothschild pointed out that a theorem of Trèves [10] implies this corollary for the sublaplacians of the (probably strictly) larger class of step- 2 nilpotent groups characterized only by

$$
\operatorname{ad}_{x}: \mathfrak{n} \rightarrow \mathfrak{z} \text { surjective for } x \notin z .
$$

That this condition is necessary for analytic hypoellipticity also follows (reviewer's remark) from arguments of Folland [5]. (Of course, one no longer expects to have a formula like that of Theorem 1 for these groups.) In this respect it is interesting to notice that one can replace condition $(\mathrm{H})$ by $(18)$ in Corollary 1 as well. Indeed, if $m<\rho(n-m)$, this corollary already gives the existence of a Lie algebra satisfying (18); on the other hand, if it is not hard to see that under this surjectivity 
assumption the maps $\operatorname{ad}_{x}: \mathfrak{n} \rightarrow \mathfrak{z}(x \notin z)$ yield global $m$-frame fields on the sphere $S^{n-m-1}(\subset \mathfrak{n} / z)$ implying, by the theorem of Adams [1], the condition of the corollary.

\section{REFERENCES}

1. J. F. Adams, Vector fields on spheres, Ann. of Math. (2) 75 (1962), 603-632.

2. M. Atiyah, R. Bott and A. Shapiro, Clifford modules, Topology 3 (1964), 3-38.

3. B. Eckmann, Beweis des Satzes von Hurwitz-Radon, Comment. Math. Helv. 15 (1942), 358-366.

4. G. Folland, A fundamental solution for a subelliptic operator, Bull. Amer. Math. Soc. 79 (1973), 373-376.

5. , On the Rothschild-Stein Lifting Theorem, Comm. Partial Differential Equations 2 (1977), $165-191$.

6. L. Hörmander, Hypoelliptic second order differential equations, Acta Math. 119 (1967), 147-171.

7. A. Kaplan and R. Putz, Boundary behavior of harmonic forms on a rank-one symmetric space, Trans Amer. Math. Soc. 231 (1977), 369-384.

8. T. Y. Lam, The algebraic theory of quadratic forms, Benjamin, New York, 1973.

9. L. Rothschild and E. Stein, Hypoelliptic differential operators and nilpotent groups, Acta Math. 137 (1976), 247-320.

10. F. Trèves, Analytic hypoellipticity of a class of pseudodifferential operators with double characteristics and applications to the $\bar{\partial}$-Neumann problem, Comm. Partial Differential Equations 3 (1978), 475-642.

Department of Mathematics, University of Massachusetts, AmHerst, MassachusetTs 01003 